\title{
Cognitive Radio Spectrum Allocation Strategy Based on Improved Genetic Algorithm
}

\author{
Bin Hou, Yunxiao Zu, Weihai Li, Gang Liu, Junjie Ding \\ School of Electronic Engineering, Beijing University of Post \& Telecommunication, Beijing China \\ Email: robinhou@163.com
}

Received March, 2013

\begin{abstract}
With the rapid development of wireless communication industry, shortage situation of spectrum resource is increasingly significant. It has become an important topic to study cognitive radio spectrum allocation algorithm that is of higher spectrum utilization ratio, less system power consumption and better algorithm efficiency. Analyzes spectrum allocation models based on genetic algorithm, and then puts forward new improved genetic algorithm. The algorithm adopts niche crowding operation to avoid individual inbreeding. It adaptively adjusts crossover and mutation probability to keep them always in the appropriate state. It provides more equal individual competition opportunity by hierarchical measures, which can effectively avert premature convergence to local optimal solution. It obviously improves the district's total transfer rate on the premise that it has met the requirements of minimum user transfer rate and limitations of maximum total power and maximum bit error rate. Simulation results prove the effectiveness of the proposed algorithm.
\end{abstract}

Keywords: Cognitive Radio; Spectrum Allocation; Genetic Algorithm; Niche; Adaption

\section{Introduction}

Under the background of increasingly shortage of spectrum resource, realization of rational allocation for cross band spectrum and utilized technology of cognitive radio (CR) are becoming focus of research in wireless communication arhjyjea. Though cognitive radio has achieved high attention from researchers, many key technology problems remain to be solved and there is still a long way to go for commercial application.

Due to the fact that CR networks are made up of plentiful high density distribution of base stations, sensor nodes and users, resource allocation should take fully into account parameters such as free carrier quantity, rate needs of users, channel quality, bit error rate constraints and transmission power, etc. But with the growth of users and subcarrier number, complexity of resource allocation problems in cognitive radio networks is increasing exponentially. Traditional resource allocation methods can no longer meet demand [1]. Besides, Equipment power, bit error rates and computational complexity of cognitive radio network are restricted owing to its properties. In this case, better resource allocation methods are required to ensure transmission quality and network performance and improve network capacity and transfer rate of cognitive radio network.

This paper puts forward a kind of cognitive radio spectrum allocation strategy based on improved genetic algorithm. The simulation results indicate that under the circumstances of same system total power, minimum user rate and bit error rate, this proposed allocation algorithm is superior to traditional common genetic algorithm on spectrum efficiency.

\section{Improved Genetic Algorithm}

Genetic Algorithm (GA) is a kind of algorithm that imitates biological evolution mechanism to search global optimal solution to target problem. It puts the potential solution in problem space as a biological individual, and encodes the individual. It imitates biological evolution process, carries out selection, crossover and mutation operation circularly and finally chooses the individual of optimal performance as the solution to a problem [2].

Genetic algorithm is appropriate to large and complex search problems, and cognitive radio spectrum allocation is such a problem - when allocating spectrum, there is an enormous quantity of available distribution schemes. It's unpractical for enumerative search, and input parameters influence each other. Thus, genetic algorithm can be applied to research on cognitive radio spectrum allocation issue.

Consider problems existing in traditional genetic algorithm as follows: (a) towards the end of algorithm running, individual genetic is approximate and their offspring cause inbreeding, thus resulting in population genetic redundancy. (b) Crossover and mutation probability 
is fixed, which influences algorithm speed. (c) The population is single. If there exists some individual whose fitness is more outstanding in the initial population, then it's easy to converge to the local optimal solution $[2,5]$. The shortage of the traditional genetic algorithm is modified and improved in this paper.

In view of problem (a), adopt niche crowding operation to remove individuals that have similar gene.

In view of problem (b), adaptively adjust crossover and mutation probability to keep them always in the optimal range.

In view of problem (c), take stratified measures to divide the population into more child population. Independently run low-level genetic algorithm in each child population. When running up to certain algebra, merge the child population and then turn to high-level genetic algorithm. Thereby reduce occurrence probability of local optimum solution.

\section{Spectrum Allocation Model Design}

\subsection{Scene Design}

Suppose a cell cellular radius is $1 \mathrm{~km}$ and a base station tower' height is $40 \mathrm{~m}$. Apply shadow fading model and supposes that shadow effect doesn't change with time. The channel gain of user $\mathrm{m}$ at subcarrier $\mathrm{n}$ is $b_{m, n} \mathrm{~dB}$. The average power spectral density of WGN (white Gaussian noise) at the receiving end is $N_{0} \mathrm{~W} / \mathrm{Hz}$. According to empirical value, set the value of $N_{0}$ to 10-12 $\mathrm{W} / \mathrm{Hz}$ [2]. The number of available subcarriers in a cell and total users is 2000 and 50. Every subcarrier bandwidth is $30 \mathrm{KHz}$. Descending frequency band is 1900$1930 \mathrm{MHz}$ and ascending frequency band is 1950-1980 MHz. Adopt MQAM (Multiple Quadrature Amplitude Modulation) modulation and permissible highest modulation order is 256. The MQAM modulation order and transmission power of user $\mathrm{m}$ at subcarrier $\mathrm{n}$ are $W_{m, n}$ and $P_{m, n}$. Bit period $T_{m, n}$ equals to 100us. If a subcarrier has not been used by any user, then set $W_{m, n}$ to 0 . Tolerable maximum BER (bit error rate) of every user is $P_{e}$, it set to $0.0005 \%$. Minimum rate demand $R_{\text {min }}$ equals to $64 \mathrm{Kbits} / \mathrm{s}$. Maximum transmission power $G_{\max }$ of base station in a cell is $80 \mathrm{~W}$.

\subsection{Channel Gain Matrix Design}

Suppose the channel gain of user $m$ at subcarrier $n$ is $b_{m, n} \mathrm{~dB}$. It is presented by matrix B.

$$
B=\left[\begin{array}{ccccc}
b_{1,1} & b_{1,2} & \cdots & b_{1, N-1} & b_{1, N} \\
b_{2,1} & b_{2,2} & \cdots & b_{2, N-1} & b_{2, N} \\
\vdots & & b_{m, n} & & \vdots \\
b_{M-1,1} & b_{M-1,2} & \cdots & b_{M-1, N-1} & b_{M-1, N} \\
b_{M, 1} & b_{M, 1} & \cdots & b_{M, N-1} & b_{M, N}
\end{array}\right]
$$

Formula (2) is for path loss value of free space[3].

$$
L_{(\mathrm{dB})}=32.45+10 \lg f+20 \lg d
$$

In the formula, $f$ represents the center frequency of carrier and $d$ represents the distance $(\mathrm{km})$ between receiving antenna and transmitting antenna.

Considering shadow fading effect, channel gain is expressed as Formula (3) [3].

$$
b_{m, n}=L(\mathrm{~dB})+\Omega_{\mathrm{s}(\mathrm{dB})}
$$

In the formula, $\Omega_{s}$ is level variation produced by shadow effect. When under simulation, assume that $\Omega_{s}$ is a normal distribution random variable with $0 \mathrm{~dB}$ mean. In macro-cellular, the standard deviation $\sigma$ ranges from $5 \mathrm{~dB}$ to $12 \mathrm{~dB}$. In the micro-cellular, the standard deviation $\sigma$ ranges from $4 \mathrm{~dB}$ to $13 \mathrm{~dB}$. The typical value of $\sigma$ is $8 \mathrm{~dB}$ and it is set to $8 \mathrm{~dB}$ for simulation.

\subsection{Target of Resource Allocation Solution}

The target of CR resource allocation discussed in this paper is described as follows. On the basis of channel gain of each subcarrier, determine which subcarrier be allocated to which users, transmission power of each subcarrier and modulation order of MQAM to achieve total maximum rate of the $\mathrm{CR}$ system. In addition, the following constraint conditions should be satisfied. 1) The transfer rate of each user should be higher than minimum rate $R_{\min }$ Mbits/s, as high as possible. 2) BER of each user is lower than the maximum BER $P_{e}$, as low as possible. 3) The system total power consumption is less than power consumption upper limit $G_{\max } \mathrm{dBm}$, as little as possible.

\subsection{Fitness Function Design}

According to the target of resource allocation, designed fitness function is delivered by formula (4):

$$
\begin{aligned}
& f_{A}(A)=D \sum_{n=1}^{N} \sum_{m=1}^{M} S_{m, n} \\
& S_{m, n} \approx \frac{\log _{2} W_{m, n}}{2}
\end{aligned}
$$

$f_{A}(A)$ stands for fitness of resource allocation scheme A. $D$ is bandwidth of subcarrier. $S_{m, n}$ Represents spectrum utilization and $M_{m, n}$ represents MQAM modulation order of user $m$ at subcarrier $n$. Therefore, the value of fitness function is system total transfer rate.

The system should meet the three constraints described in 3.3. If anyone of them is not satisfied, then set the fitness function value of the individual to 0 , resulting that it cannot participate in the generation of next population. Hence we can get individual fitness function modified from constraints. 


$$
\begin{gathered}
f_{A}(A) \\
=\left\{\begin{array}{cc}
D \sum_{n=1}^{N} \sum_{m=1}^{M} S_{m, n},\left(D \sum_{n=1}^{N} S_{m, n} \geq R_{\min }, P_{m} \leq P_{e}, g(A, B) \leq G_{\max }\right) \\
0, & \text { Others }
\end{array}\right.
\end{gathered}
$$

\section{CR Resource Allocation Based on Improved Genetic Algorithm}

Main steps of CR spectrum allocation based on improved genetic algorithm are individual coding, child population division, niche crowding operation, excluding individuals that don't accord with constraint conditions, selection, crossover and mutation of low-level genetic algorithm, adaptive adjustment of low-level algorithm parameters, selection, crossover and mutation of high-level genetic algorithm and adaptive adjustment of high-level algorithm parameters.

\subsection{Individual Coding and Child Population Division}

Formula (6) displays matrix coding form adopted by individuals.

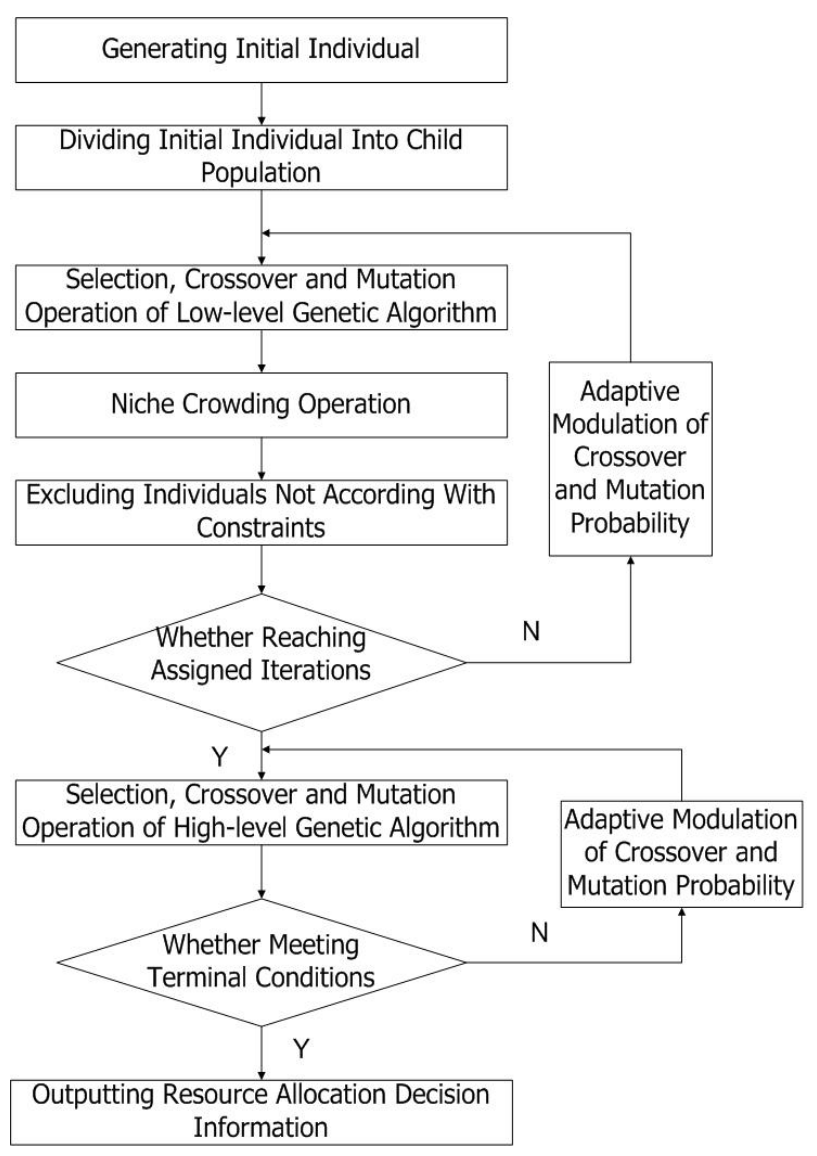

Figure 1. CR Spectrum Allocation Flow Chart Based on Improved Genetic Algorithm.

$$
\begin{aligned}
& A=\left[\begin{array}{ccccc}
a_{1,1} & a_{1,2} & \cdots & a_{1, N-1} & a_{1, N} \\
a_{2,1} & a_{2,2} & \cdots & a_{2, N-1} & a_{2, N} \\
\vdots & & a_{m, n} & & \vdots \\
a_{M-1,1} & a_{M-1,2} & \cdots & a_{M-1, N-1} & a_{M-1, N} \\
a_{M, 1} & a_{M, 1} & \cdots & a_{M, N-1} & a_{M, N}
\end{array}\right] \\
& a_{m, n}=\left(\begin{array}{ll}
W_{m, n}, & P_{m, n}
\end{array}\right)
\end{aligned}
$$

Matrix A represents one kind of spectrum resource allocation mode. There are $M$ user facilities and $N$ subcarriers in CR system. $W_{m, n}$ represents MQAM modulation order and $P_{m, n}$ represents transmission power of user $m$ at subcarrier $n$ in CR system. The two parameters are randomly generated, and each subcarrier can at most be occupied by one user facility. There are only six modulation modes of every subcarrier. The items in expression: $W_{m, n} \in\{0,16,32,64,128,256\}$ are on behalf of no use, using 16QAM, 32QAM, 64QAM, 128QAM and 256QAM modulation.

When the algorithm begins running, first it randomly generates $X \times Y$ initial individuals with matrix $A$ format. They constitute the initial population $P$ the algorithm. Then $P$ divided into $X$ child population

$$
\left[P_{1}, P_{2} \cdots P_{X-1}, P_{X}\right]^{\prime}
$$

by line. Every child population $P_{i}$ includes $Y$ individuals.

\subsection{Selection, Crossover and Mutation Operation of Low-level Genetic Algorithm}

Low-level genetic algorithm is meant for child population. When individual fitness of each resource allocation way is figured out, the probability of the $i$-th individual $A_{x, i}$ being selected in child population

$$
P_{x}=\left[A_{x, 1}, A_{x, 2} \cdots A_{x, Y}\right]
$$

is determined by the following formula[3].

$$
p_{\text {select-low }}\left(A_{x, i}\right)=\frac{f_{A}\left(A_{x, i}\right)}{\sum_{y=1}^{Y} f_{A}\left(A_{x, y}\right)}
$$

$f_{A}\left(A_{x, i}\right)$ stands for the $i$-th individual fitness of child population $P_{x}$. Operate on child population $P_{x}$ for roulette selection based on fitness. The better fitness the individual of child population owns, the larger probability the individual is selected. Then new child population can form.

After selection operation, do crossover operation on new population. The crossover operation of low-level genetic algorithm uses single-point crossover. Cross position is selected by roulette wheel selection. Mutation operation of low-level genetic algorithm adopts two-line exchange form, that is, randomly interchange individuals 
from two lines of selected matrix, as shown in Figure 2.

\subsection{Niche Crowding Operation of Low-level Genetic Algorithm}

Blend the new formed child population after selection, crossover and mutation operation with the initial noniterated child population, forming new child population with $2 \times Y$ individuals. Make use of formula (8) to compute the hamming distance between every two individuals.

$$
\left\|A_{i}-A_{j}\right\|=\sqrt{\sum_{k=1}^{M}\left(a_{i k}-a_{j k}\right)^{2}}\left(\begin{array}{c}
i=1,2, \cdots, M+N-1 \\
j=i+1, i+2, \cdots, M+N
\end{array}\right)
$$

When $\left\|A_{i}-A_{j}\right\|<L$, impose Penalty Function on individual with lower fitness between $A_{i}$ and $A_{j}$ to reduce this individual's fitness. $L$ is the minimum hamming distance limit between two individuals, whose value is $1 / 3$ of average hamming distance of the population. Rank the child population individuals in descending order depending on new fitness, and then choose the first $Y$ individuals to compose new population [4].

\subsection{Adaptive Adjustment of Crossover and Mutation Probability}

Formula (9) reveals crossover probability.

$$
F_{\text {cross-low }}\left(A_{s, i}, A_{s, j}\right)
$$

is the crossover probability of individual $A_{s, i}$ and $A_{s, j}$ in population $P_{s} . k_{1}$ is constant coefficient , set to 0.1 on the basis of empirical value. $f_{A}\left(A_{s, i}\right)$ and $f_{A}\left(A_{s, j}\right)$ is the fitness of crossover individuals. $f_{A \max }$ is the maximum fitness in $P_{s}$, and $f_{A \min }$ is the minimum fitness.

$$
F_{\text {cross-low }}\left(A_{s, i}, A_{s, j}\right)=k_{1} \frac{2 f_{A \max }-f_{A}\left(A_{s, i}\right)-f_{A}\left(A_{s, j}\right)}{f_{A \max }-f_{\text {Amin }}}
$$

Formula(10) reveals mutation probability. $k_{2}$ is constant coefficient, set to 0.02 on the basis of empirical value. $f_{A}\left(A_{s, i}\right)$ is the fitness of mutation operation individuals. $f_{A \max }$ is the maximum of individual fitness in population, and $f_{A \min }$ is the minimum of individual fitness.

$$
F_{\text {cross-low }}\left(A_{s, i}\right)=k_{2} \frac{f_{A}\left(A_{s, i}\right)}{f_{A \max }-f_{A \min }}
$$

\subsection{Selection, Crossover and Mutation Operation of High-level Genetic Algorithm}

High-level genetic algorithm is meant for the total population. When low-level genetic algorithm has independently run a certain iterative algebra in each child population, merge all child population into total population $P$. Do roulette selection operation on $P$ based on fitness.
The crossover operation of high-level genetic algorithm adopts uniform crossover [6]. Every point is potential intersection. According to the $0-1$ sequence that is randomly generated and equals the number of individual DNA bits, two father populations randomly choose individuals in accordance with corresponding position of $0-1$ sequence to generate two child populations.

\section{Analysis of Simulation Results}

Simulate for 50 times, and take average results. When there are 50 users in the system and the BER limit of each user is $0.0005 \%$, Figure 3 displays the variation of system transfer rate with traditional genetic algorithm iteration times. As can be seen, system transfer rate of traditional genetic algorithm is steadily rising with iteration times, and ultimately converge to a suboptimal value. By comparison, system transfer rate of improved genetic algorithm present in this paper is rapidly rising with iteration times, and soon reaches a high level. Therefore, the system total transfer rate obtained from resource allocation scheme based on improved genetic algorithm is superior to that of traditional genetic algorithm.

$$
A=\left[\begin{array}{lll}
a_{1,1} & \cdots & a_{1, N} \\
\vdots & \cdots & \vdots \\
a_{i, 1} & \cdots & a_{i, N} \\
\vdots & \vdots & \vdots \\
a_{j, 1} & \cdots & a_{j, N} \\
\vdots & \cdots & \vdots \\
a_{M, 1} & \cdots & a_{M, N}
\end{array}\right] \stackrel{\text { mutation }}{\longrightarrow} A^{*}=\left[\begin{array}{lll}
a_{1,1} & \cdots & a_{1, N} \\
\vdots & \cdots & \vdots \\
a_{j, 1} & \cdots & a_{j, N} \\
\vdots & \vdots & \vdots \\
a_{i, 1} & \cdots & a_{i, N} \\
\vdots & \cdots & \vdots \\
a_{M, 1} & \cdots & a_{M, N}
\end{array}\right]
$$

Figure 2. Mutation operation of low-level genetic algorithm.

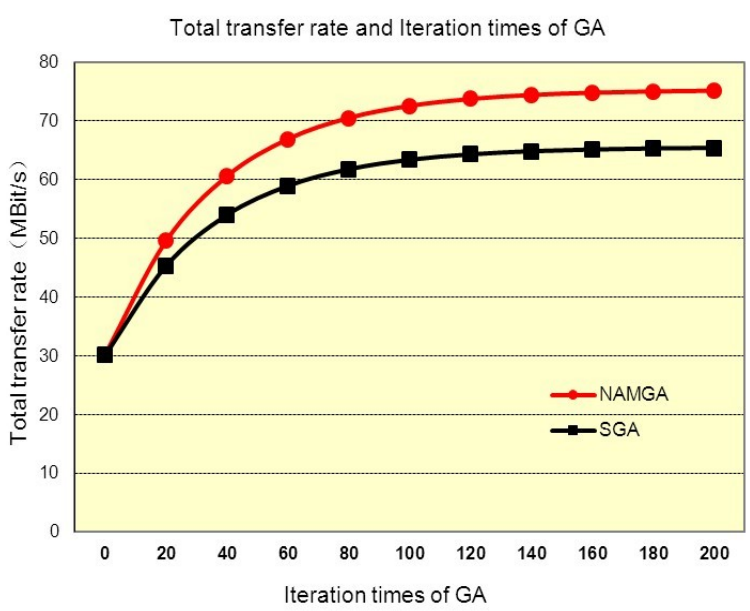

Figure 3. System Total Transfer Rate Comparison Between Improved Genetic Algorithm and Traditional Genetic Algorithm. 


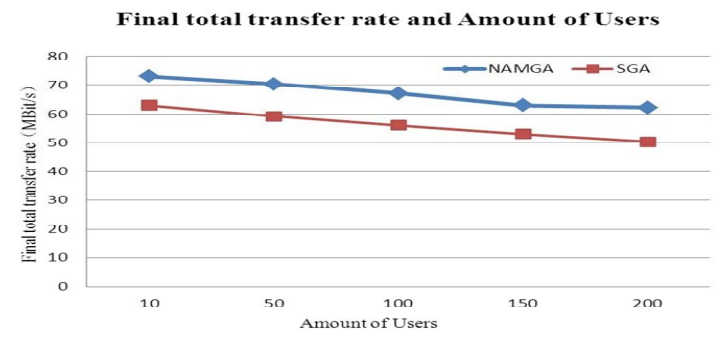

Figure 4. System Final Total Transfer Rate Variation with Amount of Users.

Figure 4 displays the variation of system final total transfer rate with amount of users when the BER limit of each user is $0.0005 \%$ and the amount of total users is 10 , $50,100,150$ or 200 . As can be seen, with increasing of users in system, the total transfer rates decline due to congestion. However, if apply the improved algorithm in this paper, all system total transfer rate obtained from resource allocation scheme increases significantly compared to traditional genetic algorithm.

\section{Conclusions}

In allusion to spectrum allocation problems in cognitive radio network, this paper comes up with resource allocation scheme based on improved genetic algorithm and constructs steps of the algorithm and related parameters. The simulation results demonstrate that in comparison to simple genetic algorithm; under the condition of the same system total power limit, minimum user rate needs and maximum BER limit, the system total transfer rate of improved genetic algorithm increases substantially.

\section{Acknowledgements}

This work was supported by the Youth Research and Innovation Program of the Electronic Engineering School, BUPT. (Grant No. 2012RC0304 ).

\section{REFERENCES}

[1] P. Kolodzy, Spectrum Policy Task Force: Findings and Recommendations, International Symposium on Advanced Radio Technologies (ISART), March 2003, pp. 1-3

[2] X. P. Wang and L. M. Cao, "Genetic Algorithm-Theory, Application and Software Implementation," Xi' an Press of Xi'an Jiaotong University, 2002, pp. 68-79.

[3] C. C. Zeng and Y. X. Zu, “Cognitive Radio Spectrum Allocation Based on Niche Adaptive Genetic Algorithm, 2011 The IET International Conference on Communication Technology and Application, Beijing, 2011, pp. 1-4.

[4] B. Wild and K. Ramchandran, "Detecting Primary Receivers for Cognitive Radio Applications. in Proc. IEEE Symp. New Frontiers Dynamic Spectrum Access Networks, 2005, pp. 124-130.

[5] R. Tandra, Fundamental Limits on Detection in Low SNR. International Conference on Wireless Networks, Communications and Mobile Computing, 2005, pp. 464-469.

[6] Y. X. Zu and J. Zhou, Cross-layer Resource Allocation and Packet Scheduling Scheme in Cognitive Radio Network, Invention Patent of People's Republic of China, 2011, pp. 1-4. 